\title{
Classical Philadelphia-negative myeloproliferative neoplasms: focus on mutations and JAK2 inhibitors
}

\author{
Grzegorz Helbig ${ }^{1}$ (D)
}

Received: 28 June 2018 / Accepted: 31 July 2018 / Published online: 3 August 2018

(c) The Author(s) 2018

\begin{abstract}
Classical Philadelphia- negative myeloproliferative neoplasms (MPNs) encompass three main myeloid malignancies: polycythemia vera (PV), essential thrombocythemia (ET), and myelofibrosis (MF). Phenotype-driver mutations in Janus kinase 2 (JAK2), calreticulin (CALR), and myeloproliferative leukemia virus oncogene (MPL) genes are mutually exclusive and occur with a variable frequency. Driver mutations influence disease phenotype and prognosis. PV patients with JAK2 exon 14 mutation do not differ in number of thrombotic events, risk of leukemic and fibrotic transformation, and overall survival to those with JAK2 exon 12 mutation. Type 2-like CALR-mutated ET patients have lower risk of thrombosis if compared with those carrying JAK2 or type 1-like CALR mutation. For ET, overall survival is comparable between patients with $J A K 2$ and either type 1-like and type 2-like CALR mutations. For MF, better OS is demonstrated for patients harboring a type 1-like CALR mutation than those with type 2-like CALR or JAK2. The discovery of driver mutations in MPNs has prompted the development of molecularly targeted therapy. Among JAK2 inhibitors, ruxolitinib (RUX) has been approved for (1) treatment of intermediate-2 and high-risk MF and (2) PV patients who are resistant to or intolerant to hydroxyurea. RUX reduces spleen size and alleviates disease symptoms in a proportion of MF patients. RUX in MF leads to prolonged survival and reduces risk of death. RUX controls hematocrit, reduces spleen size and alleviates symptoms in PV. Adverse events of RUX are moderate, however, its long-term use may be associated with opportunistic infections. Trials with other JAK2 inhibitors are ongoing.
\end{abstract}

Keywords Driver mutations · Non-driver mutations $\cdot$ Myelofibrosis $\cdot$ Essential thrombocythemia $\cdot$ Polycythemia vera $\cdot$ JAK2 inhibitors $\cdot$ Ruxolitinib

\section{Driver mutations in MPNs}

The classical Philadelphia ( $\mathrm{Ph})$-negative myeloproliferative neoplasms (MPNs) encompass three main myeloid malignancies: polycythemia vera (PV), essential thrombocythemia (ET), and myelofibrosis (MF) [1]. Of note is, that phenotype-driver mutations in Janus kinase 2 (JAK2), calreticulin (CALR), and myeloproliferative leukemia virus oncogene (MPL) genes are mutually exclusive and occur with a variable frequency in patients with classical MPNs. Approximately $97 \%$ of PV patients carry a V617F mutation

Grzegorz Helbig

ghelbig@o2.pl

1 Department of Hematology and Bone Marrow Transplantation, School of Medicine in Katowice, Medical University of Silesia, Dąbrowski street 25, 40-032 Katowice, Poland located in exon 14 of the JAK2 gene. Those PV patients who are negative for $\mathrm{V} 617 \mathrm{~F}$, may harbor mutation in exon 12. JAK2 $\mathrm{V} 617 \mathrm{~F}$ mutation is also detected in $50-60 \%$ of ET and MF patients. About 20-25\% of the patients with ET/MF harbor $C A L R$ mutation (exon 9) whereas mutation in exon 10 of the MPL gene is demonstrated in less than $10 \%$ of ET/MF cases. About $10 \%$ of either ET or MF patients are negative for all those three driver mutations [2].

In normal subjects, activation of JAK-STAT (the Janus kinase/signal transducers and activators of transcription) pathway is a consequence of ligand binding (e.g., erythropoietin) to cytokine receptors that leads to JAK proteins phosphorylation. The phosphorylated JAK proteins attract and phosphorylate STAT proteins which dimerize and enter the nucleus triggering expression of target genes causing cell growth [3]. The underlying mechanism by which driver mutations lead to myeloid proliferation results from cytokine-independent activation of JAK-STAT signaling 
pathway. All these three mutations have a gain-of-function effect on JAK-STAT signaling and are sufficient to induce myeloproliferative phenotype in mice models [4-7].

\section{Clinical correlates of driver and non-driver mutations}

Driver mutations may have an impact on disease phenotype and prognosis. PV patients with JAK2 exon 14 mutation do not differ in the number of thrombotic events, risk of leukemic and fibrotic transformation, and overall survival to those with JAK2 exon 12 mutation [8]. Interestingly, a dozen different variants of $C A L R$ exon 9 mutations have been identified, but a 52-bp deletion (type 1) and a 5-bp insertion (type 2) are the most common. Type 2-like CALR-mutated ET patients are younger and have lower risk of thrombosis despite higher platelet count if compared with those carrying $J A K 2$ or type 1-like CALR mutation. The latter mutation is associated with higher risk of fibrotic transformation. JAK2mutated MF patients are older and have lower platelet count when compared with CALR-mutated population. No difference in clinical features and risk of leukemic transformation (LT) is observed between ET and MF patients with type 1-like and type 2-like CALR mutations. ET patients carrying $J A K 2$ have highest risk of thrombosis. For ET, overall survival (OS) is comparable between patients with JAK2 and either type 1-like and type 2-like CALR mutations. For $\mathrm{MF}$, better OS is demonstrated for patients harboring a type 1-like CALR mutation than those with type 2-like CALR or $J A K 2$ [9]. MPL-mutated ET patients have lower hemoglobin levels and higher platelet count if compared with those without this mutation. The presence of $M P L$ mutation is associated with a significant risk of vascular complications [10].

Recent studies have identified several non-driver mutations which have been shown to have a prognostic impact in patients with MPNs independent of well-known conventional risk factors. Of note is, that these additional mutations are not restricted to MPNs and can be detected in other myeloid malignancies [11]. The frequency and prognostic significance of other than JAK2/CALR/MPL mutations in PV/ET patients have been reported by Mayo Group. More than 50\% of PV and ET patients were found to have at least 1 mutation other than well-described driver mutations and TET2 and $A S X L 1$ were the most common. It was demonstrated that $A S X L 1$, SRSF2, and $I D H 2$ for PV and $S H 2 B 3, S F 3 B 1$, $U 2 A F 1, T P 53, I D H 2$, and $E Z H 2$ for ET were associated with inferior survival, higher risk of leukemic, and fibrotic transformation. Of note is that the number of mutations does not carry prognostic significance [12]. For MF cohort, the presence of ASXL1, SRSF2, IDH 1/2, and EZH2 mutations was found to have a negative impact on overall survival, but only ASXL1 mutation remained significant independent of the well-validated dynamic international prognostic scoring system (DIPSS-plus) [13]. Unlike to what has been demonstrated in PV/ET, the number of these mutations negatively affected OS and leukemia-free survival [14]. A prognostic model based on the presence of high-risk molecular markers enables risk stratification for transplant-eligible MF patients [15]. The frequency and main clinical findings of commonly seen mutations in classical MPNs are presented in Table 1.

\section{Treatment of MPNs}

Currently available therapies for PV and ET do not alter the natural history of diseases and are indicated to prevent thrombotic complications. Of note is, that cytoreductive treatment should be reserved for patients who have highrisk of thrombosis-e.g., for those who are $>60$ years and/ or have history of thrombosis. For MF patients, allogeneic stem cell transplantation (SCT) remains the only curative option, but due to high-risk of transplant-related mortality, it should be proposed after careful risk/benefit assessment. Unlike SCT, the other therapeutic options alleviate disease symptoms rather than modify disease course [16].

\section{JAK2 inhibitors in MF}

\section{Ruxolitinib}

The discovery of driver mutations in MPNs has prompted the development of molecularly targeted therapy. JAK2 inhibitors acting on JAK-STAT signaling cascade lead to increased cell apoptosis and reduction of cell proliferation [17]. Among JAK2 inhibitors, ruxolitinib (RUX)-dual JAK1/ JAK2 inhibitor has been approved for the treatment of intermediate-2 and high-risk MF [18].

The primary end point of all MF trials was a reduction of $35 \%$ or more in spleen volume (SVR) from baseline at 24 or 48 weeks depending on study. The secondary end points included time to a SVR, progression-free survival (PFS), leukemia-free survival (LFS), and overall survival (OS). There have been COMFORT- 1 and COMFORT-2 studies which evaluated the efficacy and safety of RUX in MF patients. In COMFORT-1 trial, treatment with RUX resulted in SVR $\geq 35 \%$ at 24 weeks in $42 \%$ of treated patients if compared with less than $1 \%$ in placebo group. In COMFORT-2 trial, SVR $\geq 35 \%$ at week 48 was noted for $28 \%$ of patients receiving RUX vs $0 \%$ in those who received best available therapy (BAT). Spleen reduction was maintained for more than 3 years in RUX patients either in COMFORT-1 and COMFORT-2 trials. The pooled-analysis of both studies has shown that the risk of death was reduced by $30 \%$ in RUX group if compared with the control. Moreover, patients who were originally randomized to RUX survived longer than 
Table 1 Mutational frequency and main clinical findings of mutations in classic $\mathrm{Ph}(-) \mathrm{MPNs}$

\begin{tabular}{|c|c|c|c|c|}
\hline & \multicolumn{3}{|c|}{ Mutational frequency $\%$} & \multirow[t]{2}{*}{ Main clinical findings } \\
\hline & PV & ET & MF & \\
\hline \multicolumn{5}{|l|}{ Driver mutations } \\
\hline$J A K 2 \mathrm{~V} 617 \mathrm{~F}$ & 97 & 55 & 60 & JAK2V617F: $\uparrow$ age, \\
\hline$J A K 2$ exon 12 & 3 & - & - & $\uparrow \mathrm{Hgb}, \uparrow \mathrm{WBC}, \downarrow \mathrm{PLT}$ \\
\hline$C A L R$ & - & $\begin{array}{l}25 \\
\text { Type-1 } \\
(57 \%) \\
\text { Type-2 } \\
(39 \%) \\
\text { Other } \\
(4 \%)\end{array}$ & $\begin{array}{l}25 \\
\text { Type-1 }(72 \%) \\
\text { Type-2 } \\
(16 \%) \\
\text { Other } \\
(12 \%)\end{array}$ & $\begin{array}{l}\text { CALR: } \downarrow \text { age, } \uparrow \text { PLT, } \\
\downarrow \text { Hgb, } \downarrow \text { WBC in ET } \\
\text { CALR: } \downarrow \text { age, } \uparrow \text { PLT, } \\
\downarrow \text { frequency of ane- } \\
\text { mia and } \downarrow \text { leukocyto- } \\
\text { sis in MF } \\
\text { No difference in OS } \\
\text { between } J A K 2 \\
\text { V617F and JAK2 } \\
\text { exon } 12 \text { in PV } \\
J A K 2 \text { V617F increases } \\
\text { risk of thrombosis } \\
\text { in ET } \\
C A L R \text { type-1: } \uparrow \text { risk of } \\
\text { fibrotic transforma- } \\
\text { tion in ET } \\
C A L R \text { type-2: } \downarrow \text { risk of } \\
\text { thrombosis in ET } \\
\text { no difference in OS } \\
\text { and LT between } \\
C A L R \text { and } J A K 2 \\
\text { V617F in ET } \\
\text { better OS in } C A L R \\
\text { type-1 vs } C A L R \\
\text { type-2, JAK2 V617F } \\
\text { and MPL in MF } \\
\text { no difference in LT } \\
\text { rates between CALR } \\
\text { and } J A K 2 \text { V617F } \\
\text { in MF } \\
M P L \text { associated with } \\
\text { small vessel distur- } \\
\text { bances in ET [9, } 10, \\
13,16]\end{array}$ \\
\hline$M P L$ & - & 3 & 7 & \\
\hline \multicolumn{5}{|c|}{ Non-driver mutations } \\
\hline$A S X L 1$ & 12 & 11 & 22 & $A S X L 1, S R S F 2, I D H 2$ \\
\hline$S R S F 2$ & 3 & 2 & 8 & associated with \\
\hline EZH2 & - & 3 & 5 & ASXL1, SRSF 2, EZH2, \\
\hline$I D H 1 / 2$ & 2 & 3 & 5 & $\begin{array}{l}I D H 1 / 2 \text { negatively } \\
\text { correlated with OS } \\
\text { and PFS in MF } \\
I D H 2 \text { and } E Z H 2 \text { asso- } \\
\text { ciated with inferior } \\
\text { OS in ET }[3,11-13]\end{array}$ \\
\hline
\end{tabular}

ET essential thrombocythemia, $H g b$ hemoglobin, $L T$ leukemic transformation, $M F$ myelofibrosis, $O S$ overall survival, $P L T$ platelets, $P V$ polycythemia vera, $W B C$ white blood cell

those who crossed over from placebo/BAT to RUX [18-20]. The COMFORT trials have demonstrated that larger spleen size at baseline was associated with higher risk of death whereas spleen reduction on RUX resulted in better survival. Of note is, that patients with at least $25 \%$ spleen reduction on RUX had prolonged survival if compared with those without change in spleen volume [20,21]. Moreover, it has been shown that RUX alleviated disease symptoms improving patient's quality of life. An increased inflammatory responses have been demonstrated in patients with MPNs as a consequence of activation of a variety of cytokines. RUX was found to decrease serum cytokine levels reversing the constitutional symptoms thereby leading to clinical improvement [20-22]. These cytokines are produced by the mutated and non-mutated hematopoietic cells leading to progressive bone marrow fibrosis. Moreover, they seem to play a role in development of extramedullar hematopoiesis [23]. Interestingly, RUX treatment led to reduction of bone marrow fibrosis in $16 \%$ of MF patients in COMFORT-II trial [20]. MD Anderson group has reported on the results of bone marrow biopsies at baseline and at 24, 48, and 60 months of RUX. Improvement of fibrosis was demonstrated in $15 \%$, $34 \%$, and $36 \%$, respectively. These results have proved that JAK inhibition modulates the cellular components of bone marrow microenvironment which are thought to be related to fibrosis [24]. Of note is, that only three cases with complete resolution of bone marrow fibrosis on RUX have been reported so far [25-27]. Long-term treatment with RUX is associated with opportunistic infections [28].

It was demonstrated that RUX altered the JAK2 V617F allele burden in JAK2-positive patients with MF. Moreover, the decreases of allele burden correlated with spleen volume reduction and were inversely associated with disease duration. Only $11 \%$ of analyzed JAK2-positive MF patients achieved partial (PMR) or complete molecular response (CMR). Median time to PMR and CMR was 22 and 27 months, respectively. Interestingly, the spleen responses were also demonstrated in patients without allele burden reduction or in those who were JAK2- negative at baseline. Moreover, changes in allele burden did not correlate with improvement of blood parameters, clinical symptoms and bone marrow histopathology [29].

Treatment with RUX in MF can be associated with prolonged survival and reduced risk of death independent of mutation profile. Data from COMFORT-II trial have clearly demonstrated that RUX abrogated the negative impact of high-risk molecular markers. Of note is that these detrimental mutations did not decrease the probability of obtaining spleen reduction or improving clinical symptoms on RUX [30].

Anemia and thrombocytopenia are dose-dependent effects of RUX therapy. Grade 3/4 anemia and thrombocytopenia occurred in $45 \%$ and $13 \%$ of patients, respectively. One should keep in mind that RUX leads to drop in hemoglobin levels and platelet counts over the first 12 weeks of therapy with subsequent recovery. These RUX-related events require dose reductions or brief treatment interruptions as well as red blood cell or platelet transfusions [31]. Interestingly, the occurrence of RUX-induced anemia does not affect survival 
[32]. Non-hematologic side effects of RUX are mild and manageable. Of note is that two cases of secondary acute myeloid leukemia were reported in patients receiving RUX [31].

However, it should be noted, that data on RUX efficacy and safety for MF patients provided by Cochrane Systemic Review were not so evident as those quoted above. There was very low or low quality of evidence for the effect of RUX on OS and PFS when compared with placebo or BAT. Similarly, RUX did not decrease the risk of hematologic and non-hematologic toxicity when compared to comparators. The effect of RUX on reduction of spleen size remained uncertain. One should bear in mind that these conclusions were based on COMFORT-I/II industry sponsored trials and included a small number of patients [33].

\section{Momelotinib}

The safety and efficacy of momelotinib (MMB)- JAK1/2 inhibitor for intermediate and high-risk MF were evaluated in two randomized trials: SIMPLIFY-1 and SIMPLIFY-2. The former one compared MMB with RUX in patients who had not been treated with JAK2 inhibitors before. The primary study end point was similar to that of COMFORT trials. A proportion of patients who achieved $\geq 35 \%$ reduction in spleen size at 24 weeks was comparable between treated arms: $27 \%$ in MMB vs $29 \%$ in RUX. MMB was inferior when compared with RUX in terms of symptoms resolution. MMB treatment resulted in a significant decrease of transfusion requirement. Most common side effects of MMB included anemia and thrombocytopenia; $10 \%$ of treated patients developed grade $\leq 2$ peripheral neuropathy [34]. The SIMPLIFY-2 study compared MMB with BAT in MF patients who had received previous RUX. MMB was not superior to BAT (including RUX) in decreasing spleen size (7\% in MMB vs 6\% in BAT group). Adverse events were similar between groups except neuropathy which occurred in $10 \%$ of MMB-treated patients and $0 \%$ in BAT population [35]. Recently, the Mayo Group have presented their results of a 7-year follow-up of MMB treatment in MF patients who were treated between 2009 and 2010 as a part of phase $1 / 2$ study. A vast majority of patients (91\%) discontinued treatment after median of 1.4 years, the remaining 9\% are still on MMB with maximum duration of treatment exceeded 7 years. Common grade $3 / 4$ adverse events of MMB treatment included thrombocytopenia and liver enzymes increase. Clinical improvements measured as spleen and anemia responses were demonstrated for $57 \%$ of MMB-treated patients. Majority of patients improved their quality of life. The presence of ASXL1, SRSF 2 and absence of $C A L R$ type-1 mutation was associated with unfavorable survival [36].

\section{Pacritinib}

Pacritinib (PAC) is a kinase inhibitor for JAK2 and FLT3. Data from phase $1 / 2$ trial study have shown that PAC induced spleen reduction with limited hematologic toxicity [37]. The PERSIST-1 study compared the efficacy of PAC (twice and once daily) vs BAT (excluding RUX) in high-risk MF patients. At 24 week SVR $\geq 35 \%$ was achieved by $19 \%$ patients in PAC group vs $5 \%$ in BAT $(p=0.0003)$ [38]. In the PERSIST-2 study, patients with myelofibrosis and platelet count $\leq 100 \times 10^{9} / \mathrm{L}$ were randomized to PAC or BAT (including prior RUX). PAC was found to be more effective than BAT in SVR $\geq 35 \%$ (18\% vs 3\%) and led to greater than $50 \%$ reduction in total symptom score $(25 \%$ vs $14 \%$ ). Of note is, that Food and Drug Administration (FDA) in 2016 placed a full clinical hold on PAC studies following fatal complications including cerebral bleeding and cardiac failures. In January 2017 FDA has lifted its hold on PAC and a new dose-finding study PAC203 was initiated [18, 39]. Patients who benefited from PAC in PERSIST studies were allowed to continue the treatment on compassionate-use basis. Re-initiation of PAC resulted in mild improvement in SVR with low rate of hematologic toxicity. It seems reasonable to offer PAC for patients with MF and pancytopenia, especially thrombocytopenia [40].

\section{Fedratinib}

Fedratinib (FED) is a JAK2 inhibitor which efficacy was first evaluated in the JAKARTA-1 study. FED at two doses (400 $\mathrm{mg}$ and $500 \mathrm{mg}$ ) was compared with placebo in 289 patients with intermediate- 2 or high-risk MF. Spleen response was achieved in 36\% of FED-treated patients at $400 \mathrm{mg}, 40 \%$ at $500 \mathrm{mg}$ and $1 \%$ in the placebo group. Four patients receiving FED at $500 \mathrm{mg}$ daily developed Wernicke encephalopathy (WE) [41]. The JAKARTA-2 study included patients with MF who were resistant to or intolerant to RUX. Spleen response was noted in 53\% of patients who were RUX resistant and $63 \%$ of patients who were RUX intolerant. However, it should be mentioned that $7 \%$ of patients died during the study as a consequence of encephalopathy. It was the reason for study discontinuation [42]. Recently, the analysis of 9 FED trials with 670 patients has revealed that the prevalence of encephalopathy in this population is less than $1 \%$. Based on this data, FDA decided to lift the clinical hold [43].

\section{JAK2 inhibitors in PV/ET}

\section{Ruxolitinib in PV}

RUX has been approved for PV patients who are resistant to or intolerant to hydroxyurea based on a phase 3 study 
Table 2 JAK2 inhibitors in clinical trials

\begin{tabular}{lllll}
\hline Compound & Comparator & Trial & Indication & Primary end point \\
\hline RUX & PBO & COMFORT-1 [19] & MF & SVR $\geq 35 \%$ at 24 week: 42\% (RUX) vs 1\% (PBO) \\
RUX & BAT & COMFORT-2 [20] & MF & SVR $235 \%$ at 48 week: 28\% (RUX) vs 0\% (BAT) \\
MMB & RUX & SIMPLIFY-1 [34] & MF & SVR $\geq 35 \%$ at 24 week: 27\% (MMB) vs 29\% (RUX) \\
MMB & BAT (incl. RUX) & SIMPLIFY-2 [35] & MF & SVR $\geq 35 \%$ at 24 week: 7\% (MMB) vs 6\% (BAT) \\
MMB & BAT & RESPONSE [44] & PV & Hematocrit control at week 32: 60\% (RUX) vs 20\% (BAT) \\
PAC & BAT & PERSIST-1 [38] & MF & SVR $35 \%$ at 32 week: 38\% (RUX) vs 1\% (BAT) \\
PAC & BAT (incl. RUX) & PERSIST-2 [39] & MF & SVR $\geq 35 \%$ at 24 week: 19\% (PAC) vs 5\% (BAT) \\
FED & PBO & JAKARTA-1 [41] & MF & SVR $\geq 35 \%$ at 24 week: 36\% (FED400) vs 40\% (FED500) \\
& Single arm (RUX & JAKARTA-2 [42] & MF & Vs 1\% (PBO) \\
FED & resistant/intolerant) & & & SVR $25 \%$ at 24 week: 55\% \\
RUX & BAT & MAJIC-ET [47] & ET & CRR at 12 months: 47\% (RUX) vs 44\% (BAT) \\
\hline
\end{tabular}

$C R R$ complete response rate, $B A T$ best available therapy, $F E D$ fedratinib, $M M B$ momelotinib, $P B O$ placebo, $P A C$ pacritinib, $R U X$ ruxolitinib, $S V R$ spleen volume response

(RESPONSE) comparing RUX with BAT. The primary end point was both hematocrit control and $\geq 35 \%$ SVR reduction and at week 32 . Hematocrit control was observed in $60 \%$ of patients in RUX group when compared to $20 \%$ of those in BAT arm. At least a $35 \%$ SVR achieved $38 \%$ and $1 \%$ patients, respectively. There was a significant difference in complete hematologic remission (CHR) rate: $24 \%$ in RUX vs $9 \%$ in BAT. Moreover, the reduction in total symptom score was greater in RUX patients. Side effects were manageable and similar to that presented in COMFORT trials [44]. The RESPONSE trial has also focused on long-term effect of RUX treatment on JAK2V617F allele burden. JAK2-positive PV patients were randomized to RUX or BAT with cross-over to RUX at week 32. It was demonstrated that mean decrease of $J A K 2$ allele burden from baseline ranged from -12 to $-40 \%$ (RUX arm) and -6 to $-18 \%$ (RUX cross-over). CMR and PMR were demonstrated in 3 and 54 patients, respectively. However, one should bear in mind that the reduction in allele burden did not correlate with clinical outcome. These findings were consistent with those seen for MF [45]. Recently, a 4-year follow-up of RESPONSE study has been presented. The median duration of CHR was not reached and $54 \%$ of patients remained in CHR. The estimated 5-year OS rates were comparable between arms. Hematologic toxicity improved with RUX continuation as well as non-hematologic adverse events. Infection was the most common side effect of RUX. Rates of thrombotic events were low in both arms [46].

\section{Ruxolitinib in ET}

The MAJIC-ET study compared the efficacy of RUX vs BAT for ET intolerant or resistant to HU. Fifty-eight patients were randomized to RUX, however, there was no evidence of superiority of RUX when compared to BAT at 12 months [47]. In contrast, MD Anderson study has demonstrated that RUX treatment was associated with the improvement in ET-related symptoms as well as better and durable platelet, leukocyte, and hemoglobin control. The reduction of JAK2allele burden was also noted [48].

\section{Momelotinib in PV/ET}

A phase 2 trial evaluated MMB in patients with $\mathrm{PV}$ and ET, however, it was prematurely discontinued due to limited efficacy [49]. The results of studies with JAK2 inhibitors in classical MPNs are shown in Table 2.

\section{JAK2 inhibitors before stem cell transplantation}

Patients with intermediate-2 and high-risk MF remain candidates both to RUX and SCT. Currently, the majority of transplant-eligible patients are treated with RUX before SCT in daily clinical practice. Several studies reported on the outcome of SCT for MF with prior exposure to RUX [50, 51]. Among 66 patients who discontinued RUX before SCT, 2 patients required SCT delay due to serious adverse events (AEs) It was concluded that RUX should be stopped just before conditioning commencement. AEs were more common in those who discontinued treatment $\geq 6$ days before conditioning. Treatment with RUX did not negatively influence outcome after SCT [50]. There is a little experience with RUX after SCT. It was demonstrated that RUX may prevent the occurrence of acute graft vs host disease, however, $40 \%$ of transplanted patients developed cytomegalovirus reactivation [52]. 


\section{Conclusions}

Discoveries of driver and non-driver mutations in MPNs allow to simplify diagnostic algorithm as well as better define disease prognosis. They may also serve as drug targets. Current experience with JAK2 inhibitors is practically restricted to patients with intermediate and highrisk MF. These compounds alleviate disease symptoms and reduce spleen size in a proportion of patients with MF. Their impact on overall survival is debatable. RUX reduces hematocrit and spleen volume in PV patients, but was found ineffective in ET. Long-term use of RUX has been associated with opportunistic infections. SCT still remains only curative treatment for MF. Peritransplant role of RUX has to be elucidated.

\section{Compliance with ethical standards}

Conflict of interest The author disclosed no conflict of interest.

Open Access This article is distributed under the terms of the Creative Commons Attribution 4.0 International License (http://creativeco mmons.org/licenses/by/4.0/), which permits unrestricted use, distribution, and reproduction in any medium, provided you give appropriate credit to the original author(s) and the source, provide a link to the Creative Commons license, and indicate if changes were made.

\section{References}

1. Arber DA, Orazi A, Hasserjian R, et al. The 2016 revision to the World Health Organization classification of myeloid malignancies and acute leukemia. Blood. 2016;127:2391-405.

2. Nangalia J, Green TR. The evolving genomic landscape of myeloproliferative neoplasms. Hematol Am Soc Hematol Educ Program. 2014;2014:2870296.

3. Schischlik F, Kralovics R. Mutations in myeloproliferative neoplasms - their significance and clinical use. Expert Rev Hematol. 2017;10:961-73.

4. Mullally A, Lane SW, Ball B, et al. Physiological Jak2V617F expression causes a lethal myeloproliferative neoplasm with differential effects on hematopoietic stem and progenitor cells. Cancer Cell. 2010;17:584-96.

5. Li J, Kent DG, Chen E, Green AR. Mouse models of myeloproliferative neoplasms: JAK of all grades. Dis Model Mech. 2011;4:1834-9.

6. Marty $\mathrm{C}$, Pecquet $\mathrm{C}$, Nivarthi $\mathrm{H}$, et al. Calreticulin mutants in mice induce an MPL-dependent thrombocytosis with frequent progression to myelofibrosis. Blood. 2016;127:1307-16.

7. Pecquet $\mathrm{C}$, Staerk J, Chaligne R, et al. Induction of myeloproliferative disorder and myelofibrosis by thrombopoietin receptor W515 mutants is mediated by cytosolic tyrosine 112 of the receptor. Blood. 2010;115:1037-48.

8. Scott LM. The JAK2 exon 12 mutations in polycythemia vera and idiopathic erythrocytosis. Am J Hematol. 2011;86:668-76.

9. Pietra D, Rumi E, Ferretti W, et al. Differential clinical effects of different mutation subtypes in $C A L R$-mutant myeloproliferative neoplasms. Leukemia. 2016;30:431-8.
10. Vannucchi AM, Antonioli E, Guglielmelli P, et al. Characteristics and clinical correlates of MPL $515 \mathrm{E}>\mathrm{L} / \mathrm{K}$ mutation in essential thrombocythemia. Blood. 2008;112:844-7.

11. Vainchenker W, Kralovics R. Genetic basis and molecular pathophysiology of classical myeloproliferative neoplasms. Blood. 2017;129:667-79.

12. Tefferi A, Lasho TL, Guglielmelli P, et al. Targeted deep sequencing in polycythemia vera and essential thrombocythemia. Blood Adv. 2016;1:21-30.

13. Vannucchi AM, Lasho TL, Guglielmelli P, et al. Mutations and prognosis in primary myelofibrosis. Leukemia. 2013;27:1861-9.

14. Guglielmelli P, Lasho TL, Rotunno G, et al. The number of prognostically detrimental mutations and prognosis in primary myelofibrosis: an international study of 797 patients. Leukemia. 2014;28:1804-10.

15. Guglielmelli P, Lasho TL, Rotunno G, et al. MIPSS70: MutationEnhanced International Prognostic Score System for Transplantation-Age Patients with Primary Myelofibrosis. J Clin Oncol. 2018;36:310-8.

16. Tefferi A, Pardanani A. Myeloproliferative Neoplasms. A Contemporary Review. JAMA Oncol. 2015;1:97-105.

17. Quintas-Cardama A, Vaddi K, Liu P, et al. Preclinical Characterization of the selective JAK1/2 inhibitor INCB018424: therapeutic implications for the treatment of myeloproliferative neoplasms. Blood. 2010;115:3109-17.

18. Passamonti F, Maffioli M. The role of JAK2 inhibitors in MPNs 7 years after approval. Blood. 2018;131:2426-35.

19. Verstovsek S, Gotlib J, Mesa RA, et al. Long-term survival in patients treated with ruxolitinib for myelofibrosis: COMFORT-I and -II pooled analyses. J Hematol Oncol. 2017;10:156.

20. Harrison CV, Vannucchi AM, Kiladjian JJ, et al. Long-term findings from COMFORT-II, a phase of 3 study of ruxolitinib vs best available therapy for myelofibrosis: 5-year final study results. Leukemia. 2017;31:775.

21. Mondet J, Hussein K, Mossuz P. Circulating cytokine levels as markers of inflammation in Philadelphia-negative myeloproliferative neoplasms: diagnostic and prognostic interest. Mediators Inflamm. 2015;2015:670580.

22. Vannucchi AM, Kantarjian HM, Kiladjian JJ, et al. A pooled analysis of overall survival in COMFORT-I and COMFORT-II, 2 randomized phase III trials of ruxolitinib for the treatment of myelofibrosis. Haematologica. 2015;100:1139-45.

23. Vaichenker W, Kralovics R. Genetic basis and molecular pathophysiology of classical myeloproliferative neoplasms. Blood. 2017;129:667-79.

24. Kvasnicka HM, Thiele J, Bueso-Ramos CE, et al. Long-term effects of ruxolitinib versus best available therapy on bone marrow fibrosis in patients with myelofibrosis. J Hematol Oncol. 2018;11:42.

25. Wilkins BS, Radia D, Woodley C, et al. Resolution of bone marrow fibrosis in a patients receiving JAK1/JAK2 inhibitor treatment with ruxolitinib. Haematologica. 2013;98:1872-6.

26. Molica M, Serrao A, Saracino R, et al. Disappearance of fibrosis in secondary myelofibrosis after ruxolitinib treatment: new endpoint to achieve. Ann Hematol. 2014;93:1951-2.

27. Al-Ali HK, Hubert K, Lange T, et al. Complete clinical, histopathologic and molecular remission of primary myelofibrosis with long-term treatment with the JAK1/2 inhibitor ruxolitinib. Blood. 2014; $124: 1836$.

28. Lussana F, Cattaneo M, Rambaldi A, Squizatto A. Ruxolitinibassociated infections: a systemic review and meta-analysis. Am J Hematol. 2018;93:339-47.

29. Deininger M, Radich J, Burn TC, Huber R, Paranagama D, Verstovsek $\mathrm{S}$. The effect of long-term ruxolitinib treatment on $J A K 2 \mathrm{p} . \mathrm{V} 617 \mathrm{~F}$ allele burden in patients with myelofibrosis. Blood. 2015;126:1551-4. 
30. Guglielmelli P, Biamonte F, Rotunno G, et al. Impact of mutational status on outcomes in myelofibrosis patients treated with ruxolitinib in the COMFORT-II study. Blood. 2014;123:2157-60.

31. Kantarjian HM, Silver RT, Komrokji RS, Mesa RA, Tacke R, Harrison CN. Ruxolitinib for myelofibrosis - an update of its clinical effects. Clin Lymphoma Myeloma Leuk. 2013;13:638-45.

32. Gupta V, Harrison C, Hexner EU, et al. The impact of anemia on overall survival in patients with myelofibrosis treated with ruxolitinib in the COMFORT studies. Haematologica. 2016;101:e482-4.

33. Marti-Carvajal AJ, Anand V, Sola I. Janus kinase-1 and Janus kinase-2 inhibitors for treating myelofibrosis. Cochrane Database Syst Rev. 2015;10:CD010298.

34. Mesa RA, KIladjian JJ, Catalano JV, et al. SIMPLIFY-1: a phase III randomized trial of momelotinib versus ruxolitinib in janus kinase inhibitor-naive patients with myelofibrosis. J Clin Oncol. 2017;35:3844-50.

35. Harrison CN, Vannucchi AM, Platzbecker U, et al. Momelotinib vesrus best available therapy in patients with myelofibrosis previously treated with ruxolitinib (SIMPLIFY-2): a randomized, openlabel, phase 3 trial. Lancet Haematol. 2018;5:e73-81.

36. Tefferi A, Barraco D, Lasho TL, et al. Momelotinib therapy for myelofibrosis: a 7-year follow-up. Blood Cancer J. 2018;8:29.

37. Verstovsek S, Odenike O, Singer JW, Granston T, Al-Fayoumi S, Deeg HJ. Phase 1/2 study of pacritinib, a next generation JAK2/ FLT3 inhibitor, in myelofibrosis or other myeloid malignancies. J Hematol Oncol. 2016;8:137.

38. Mesa RA, Vannucchi AM, Mead A, et al. Pacritinib versus best available therapy for the treatment of myelofibrosis irrespective of baseline cytopenias (PERSIST-1): an international, randomized, phase 3 trial. Lancet Haematol. 2017;4:e225-36.

39. Mascarenhas J, Hoffman R, Talpaz M, et al. Pacritinib vs best available therapy, including ruxolitinib, in patients with myelofibrosis: a randomized clinical trial. JAMA Oncol. 2018;4:652-9.

40. Mascarenhas J, Virtgaym E, Stal M, et al. Outcomes of patients with myelofibrosis treated with compassionate use pacritinib: a sponsor-independent international study. Ann Hematol. 2018. https://doi.org/10.1007/s00277-018-3309-6.

41. Pardanani A, Harrison C, Cortes J, et al. Safety and efficacy of Fedratinib in Patients with primary or secondary myelofibrosis: a randomized clinical trial. JAMA Oncol. 2015;1:643-51.

42. Harrison CN, Schaap N, Vannucchi AM, et al. Janus kinase-2 inhibitor fedratinib in patients with myelofibrosis previously treated with ruxolitinib (JAKARTA-2): a single-arm, open-label, non-randomised, phase2, multicenter study. Lancet Haematol. 2017;4:e317-24.

43. Harrison CN, Mesa RA, Jamieson C, et al. Case series of potential Wernicke's encephalopathy in patients treated with fedratinib [abstract]. Blood. 2017;130(suppl 1):4197.

44. Vannucchi AM, Kiladjian JJ, Griesshammer M, et al. Ruxolitinib versus Standard Therapy for the Treatment of Polycythemia Vera. N Eng J Med. 2015;372:426-35.

45. Vannucchi AM, Verstovsek S, Guglielmelli P, et al. Ruxolitinib reduces JAK2p.V617F allele burden in patients with polycythemia vera enrolled in the RESPONSE study. Ann Hematol. 2017;96:1113-20.

46. Kiladjian JJ, Verstovsek S, Griesshammer M, et al. Results from the 208-week (4 year) follow-up of the RESPONSE Trial, a phase 3 study comparing ruxolitinib with best available therapy for the treatment of polycythemia vera [abstract]. Blood. 2017;130(suppl. 1):322.

47. Harrison CN, Mead AJ, Panchal A, et al. Ruxolitinib vs best available therapy for ET intolerant or resistant to hydroxycarbamide. Blood. 2017;130:1889-97.

48. Verstovsek S, Passamonti F, Rambaldi A, et al. Ruxolitinib for essential thrombocythemia refractory to or intolerant to hydroxyurea: a long-term phase 2 study results. Blood. 2017;130:1768-71.

49. Verstovsek S, Courby S, Griesshammer M, et al. A phase 2 study of momelotinib, a poten JAK1 and JAK2 inhibitor, in patients with polycythemia vera or essential thrombocythemia. Leuk Res. 2017;60:11-7.

50. Shanavas M, Popat U, Michaelis LC, et al. Outcomes of allogeneic hematopoietic cell transplantation in patients with myelofibrosis with prior exposure to janus kinase $1 / 2$ inhibitors. Biol Blood Marrow Transplant. 2016;22:432-40.

51. Kadir SSA, Christopoiet S, Gerald MW, et al. Impact of ruxolitinib pretreatment on outcomes after allogeneic stem cell transplantation in patients with myelofibrosis. Eur J Haematol. 2018. https://doi.org/10.1111/ejh.

52. Kroger N, Shahnaz Syed Abd Kadir S, Zabelina T, et al. Peritransplantation ruxolitinib prevents acute graft-versus-host disease in patients with myelofibrosis undergoing allogeneic stem cell transplantation. Biol Blood Marrow Transplant. 2018. https://doi. org/10.1016/j.bbmt.2018.05.023. 